\title{
Trichotillomania: What Do We Know So Far?
}

\author{
Daniel Fernandes Melo ${ }^{a}$ Caren dos Santos Lima ${ }^{\mathrm{b}, \mathrm{c}}$ Bianca Maria Piraccini ${ }^{\mathrm{d}} \mathrm{e}$ \\ Antonella Tosti ${ }^{\mathrm{f}}$ \\ aDermatology Department, University of State of Rio de Janeiro (UERJ), Rio de Janeiro, Brazil; bDermatology \\ Department, University of State of Pará (UEPA), Belém, Brazil; 'Dermatology Department, University Center of Pará \\ (CESUPA), Belém, Brazil; dDepartment of Experimental, Diagnostic and Specialty Medicine (DIMES) Alma Mater \\ Studiorum University of Bologna, Bologna, Italy; eIRCCS Azienda Ospedaliero-Universitaria di Bologna, Bologna,

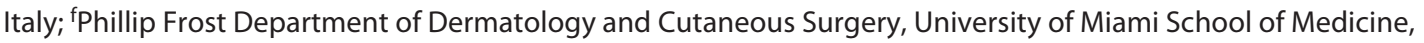 \\ Miami, FL, USA
}

\section{Keywords}

Trichotillomania - Hair pulling · Impulse-control disorder · Alopecia

\begin{abstract}
Trichotillomania is defined as an obsessive-compulsive or related disorder in which patients recurrently pull out hair from any region of their body. The disease affects mainly female patients, who often deny the habit, and it usually presents with a bizarre pattern nonscarring patchy alopecia with short hair and a negative pull test. Trichoscopy can reveal the abnormalities resulting from the stretching and fracture of hair shafts, and biopsy can be necessary if the patient or parents have difficulties in accepting the self-inflicted nature of a trichotillomania diagnosis. Trichotillomania requires a comprehensive treatment plan and interdisciplinary approach. Physicians should always have a nonjudgmental, empathic, and inviting attitude toward the patient. Behavioral therapy has been used with success in the treatment of trichotillomania, but not all patients are willing or able to
\end{abstract}

comply with this treatment strategy. Pharmacotherapy can be necessary, especially in adolescents and adult patients. Options include tricyclic antidepressants, selective serotonin reuptake inhibitors, and glutamate-modulating agents. Glutamate-modulating agents such as N-acetylcysteine are a good first-line option due to significant benefits and low risk of side effects. Physicians must emphasize that the role of psychiatry-dermatology liaison is extremely necessary with concurrent support services for the patient and parents, in case of pediatric patients. In pediatric cases, parents should be advised and thoroughly educated that negative feedback and punishment for hair pulling are not going to produce positive results. Social support is a significant pillar to successful habit reversal training; therefore, physicians must convey the importance of familial support to achieving remission. This is a review article that aims to discuss the literature on trichotillomania, addressing etiology, historical aspects, clinical and trichoscopic features, main variants, differential diagnosis, diagnostic clues, and psychological and pharmacological management.

(c) 2021 S. Karger AG, Basel karger@karger.com www.karger.com/sad
(C) 2021 S. Karger AG, Basel 


\section{Introduction}

Trichotillomania, also called hair-pulling disorder, is defined by the Diagnostic and Statistical Manual of Mental Disorders (DSM-V) as an obsessive-compulsive or related disorder in which subjects recurrently pull out hair from any region of their body, resulting in hair loss [1-3]. The psychosocial aspects of trichotillomania are greatly underestimated, but recent literature suggests an increased interest in this neglected area [2].

Although extensive epidemiological studies are lacking, the estimated prevalence data suggest that $0.5-2 \%$ of the general population suffers from this disorder [2]. However, as some people who suffer from trichotillomania feel ashamed of their condition, real prevalence may be higher [3].

Epidemiologic data in pediatric population are relatively scarce, but lifetime prevalence is estimated to be around $1-3 \%$. The most common sites of hair removal are the scalp, eyebrows, eyelashes, and pubic region [4]. Limbs, underarms, and chest hair can also be involved [2].

In adults, trichotillomania appears to have a large female preponderance, with a female-to-male ratio of 4:1 [3]. Regarding pediatric trichotillomania, the disorder most often affects female children between 9 and 13 years old, who generally deny the habit [5].

Trichotillomania is a highly comorbid disorder, with a lifetime prevalence of concomitant psychiatric disease as high as $80 \%$. The most common associated disorders are anxiety, major depression, substance misuse, eating disorders, posttraumatic stress disorder, personality disorders, and body dysmorphic disorder [6]. It has been reported to overlap with skin-picking and nail-biting [1]. Over $20 \%$ of patients may concomitantly suffer from trichophagia, when patients consume the pulled hair creating trichobezoars, or hairballs, which can lead to significant gastrointestinal complications and the need for further surgical intervention [3, 4]. A rarer presentation of the trichobezoar is the "Rapunzel syndrome," in which the tail of the hairball extends into the intestines and may cause intestinal obstruction [2]. The aim of this article is to review the literature on trichotillomania, addressing etiology, historical aspects, clinical and trichoscopic features, main variants, diagnostic clues, and management.

\section{Methods and Results}

A literature search in the scientific database MEDLINE through PubMed was performed in January 2021, using the keywords "trichotillomania" AND “alopecia," "trichotillomania" AND "hair pulling," and "trichotillomania” AND "impulse control disorder."
We limited the search to articles available in English and considered those mentioning the dermatological aspects of this condition, its trichoscopic features, diagnostic criteria, and treatment options. The references of these articles were reviewed to identify additional resources. After excluding duplicate titles, we had a total of 13 relevant articles.

\section{Discussion}

Trichotillomania has been discussed in the medical literature for over a century. Nevertheless, it was officially introduced as a mental illness in DSM in 1987, when it was classified as an impulse control disorder. DSM-V included trichotillomania in the chapter on obsessive-compulsive and related disorders, along with excoriation disorder, body dysmorphia, hoarding disorder, and obsessive-compulsive disorder [3].

The etiology of trichotillomania is not well understood yet. However, it is likely to be the result of the interaction of several factors on a single patient (genetic, psychological, social, and neurobiological) [2].

While trichotillomania may be triggered by stress, the habit itself also causes significant distress, low self-esteem, guilt, and shame. Most people report that pulling is painless, if not pleasurable. It has been proposed that hair pulling creates "counterirritation" to reduce the stress perception by the brain [2].

Two types of hair pulling have been described for trichotillomania: automatic and focused. Automatic occurs outside of one's awareness, while focused pulling happens in awareness and in response to negative emotional states, such as stress, sadness, anger, or anxiety [1]. Pulling may occur in private, leading parents to believe the hair is falling out in pediatric cases. Younger children more often fall in the automatic category; therefore, they do not recall actual pulling but may admit to "play with hair." On the other hand, older children and over $75 \%$ of adults tend to manifest focused pulling in response to stressful events $[1,4]$. Focused hair pullers tend to pull out hair that feels different from the rest (kinky, white, or odd texture), and they may also have other associated rituals such as chewing, hair licking, or trichophagia [2].

Clinically, trichotillomania presents with a diffuse or bizarre-shaped pattern, irregular nonscarring patchy alopecia (shown in Fig. 1,2) $[1,7,8]$ and can present with 1 or 2 patches or with diffuse scalp involvement. The frontoparietal region is more often affected, and eyebrows, upper eyelashes, and pubic area are also commonly involved. Less commonly, face, limbs, underarms, and chest hair are also affected. The shape of the patches is bizarre 


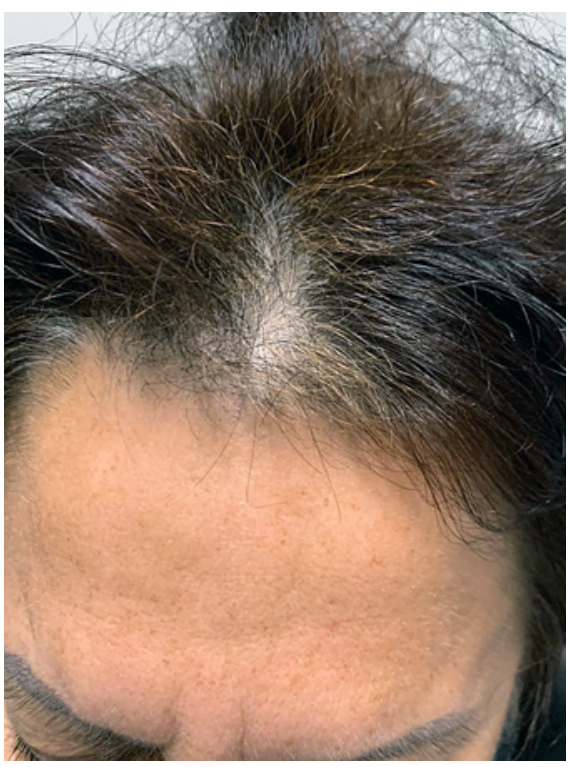

Fig. 1. Small irregular-shaped patch of hair loss in the frontal region of a 50-year-old woman diagnosed with depression and anxiety.

with angular or irregular borders. Sometimes the alopecia of the crown is surrounded by a rim of unaffected hair at the periphery, resembling tonsures of Christian monks ("Friar Truck" sign) [2, 3, 9]. The hair shafts have various lengths due to different fracture points of the hair shafts or the hair being pulled several times, producing a rough feeling when touching the scalp[7].

The differential diagnosis for trichotillomania includes alopecia areata, tinea capitis, traction alopecia, and telogen effluvium [4]. Trichoscopy reveals abnormalities resulting from the stretching and fracture of hair shafts. Common trichoscopic signs include black dots, broken hair shafts of different lengths, and yellow dots $[5,8]$.

Many other signs, all variants of broken hairs, have been described, including longitudinal split ends of hairs (short hairs with trichoptilosis), coiled hairs, flame hairs (semitransparent, wavy, and cone-shaped hair residues, resembling a fire flame), V-signs (2 or more hairs emerging from 1 follicular unit and broken at the same length), tulip hairs (short hairs with a tulip leaf-like hyperpigmentation at the distal end), and sprinkled hairs (only a sprinkled "hair powder," resulting from hair damage) $[8,9]$ (Fig. 3, 4).

Hook hairs or question mark hairs are partially coiled hairs due to a contraction of the remaining hair fixed to the scalp after the distal shaft has been pulled. They are

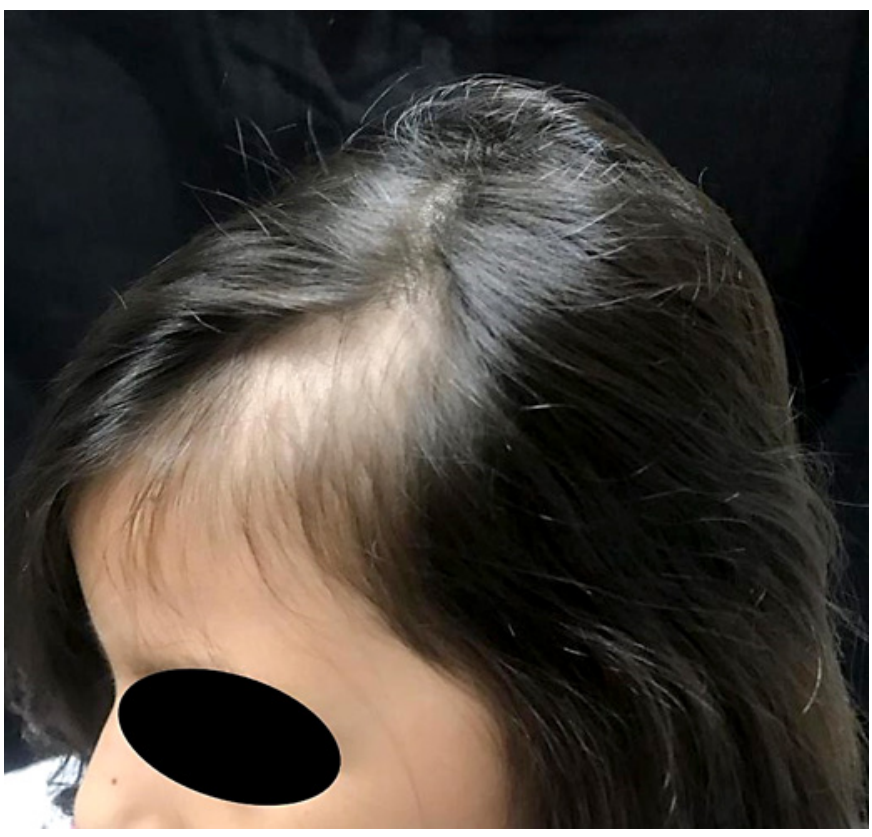

Fig. 2. A 9-year-old girl with a bizarre-shaped alopecia patch in the frontotemporal region.

considered as a highly-specific trichoscopic finding associated with trichotillomania [5].

Trichotillomania can be difficult to distinguish from alopecia areata at dermoscopy. Clinically, a negative hair pull test and absence of hypopigmented regrowing vellus hair favor a diagnosis of trichotillomania $[5,8]$.

Recently, an article reported pediatric cases of trichotillomania without patches (trichotillomania incognito), in which the patients clinically presented focal low hair density and negative pull test in the affected area associated with trichoscopic findings of trichotillomania. The absence of patches, with no specific complaint, makes the diagnosis even more challenging. In these cases, trichoscopy is essential to allow early recognition of the disease.

Trichoscopy is also useful to demonstrate the signs of plucking to the parents of children with trichotillomania $[1,8]$. Histopathology on horizontal sections shows a noninflammatory nonscarring alopecia in which the morphological changes are those of follicular damage secondary to the external insult, with distortion of the hair follicle anatomy and with perifollicular and intrafollicular hemorrhage. Additional findings include melanin pigment casts, loss of hair shafts, and trichomalacia, where the hair shaft is dysmorphic, with incomplete cornification and irregular pigmentation. The number of hair follicles is normal, with an increased occurrence of catagen 
Fig. 3. Trichoscopy of the eyelashes (a) and scalp (b) of a patient with trichotillomania presenting black dots (yellow circles), broken hairs with multiple lengths (red arrows), and V-sign (blue circle) $(\times 20)$.
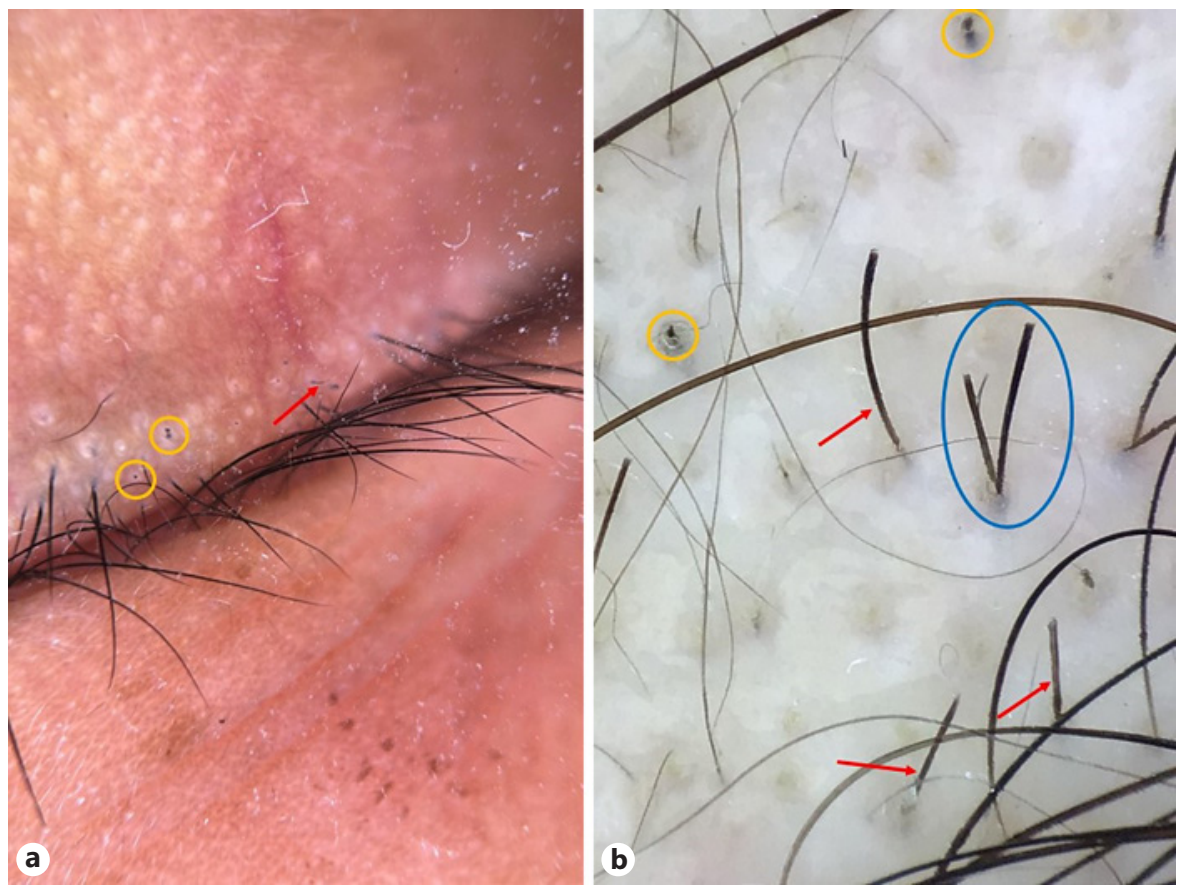

or telogen hair follicles and without significant inflammation [7].

The main variants of trichotillomania include trichoteiromania, trichotemnomania, trichodaganomania, telogen mania, and trichophobia $[10,11]$.

1. Trichoteiromania refers to the compulsive action of scratching and rubbing of the scalp, which results in fracture of the hair shafts. This is usually associated with skin dryness and itching conditions, such as lichen simplex chronicus. Clinically, it presents as single or multiple irregular patches of alopecia, with scaling, lichenification, and hair breakage $[10,12]$. Trichoscopic findings of trichoteiromania include proximal trichorrhexis nodosa and "broom hairs" which are broken hair shafts longitudinally split into 2 or 3 parts. Broken hair shaft with different lengths, perifollicular scaling, and erythema can also be seen [10].

2. Trichotemnomania is hair loss due to cutting or shaving. Patients with this condition show signs of shaving or hair-cutting in the affected areas with an otherwise healthy-appearing scalp. Trichoscopic examination of trichotemnomania may show short, broken, nonvellus hairs, without a decrease in follicle density, exclamation mark hairs, yellow dots, or black dots [10].

3. Trichodaganomania is the process of biting one's own hair on accessible sites resulting in hair loss in the affected areas. This condition shares some features with other variants of trichotillomania, including a com- pulsive need to remove hair from the affected areas followed by feelings of gratification. Due to the method of hair removal, the scalp is not involved in trichodaganomania, and affected areas are generally accessible sites such as the dorsal forearms. Microscopic features of examined hair may show a smooth blunted shaft at the bite site and a lack of attached root sheaths or hair bulbs, as would be seen in trichotillomania [10].

4. Telogen mania represents obsessive-compulsive fits of fierce hair brushing in women [11].

5. Trichophobia denotes plucking of hair based on the delusion of having to pull something out of the hair roots [11].

Although trichotillomania is considered a psychiatric diagnosis, most patients will initially present to a dermatologist for evaluation. Therefore, dermatologists should be aware of the peculiarities of this disease to provide appropriate advice and treatment for their patients [4]. The diagnostic criteria for trichotillomania are listed in Table 1 [3]. Although the course of illness may vary, trichotillomania is commonly a chronic disorder with fluctuations in intensity over time [3].

As there is currently no gold-standard treatment for trichotillomania, disease management is challenging. It consists of both psychotherapeutic and pharmacological options. Treatment recommendations may vary according to the patient's age. In prescholar children, trichotillomania is considered a habit disorder that is expected to disappear 

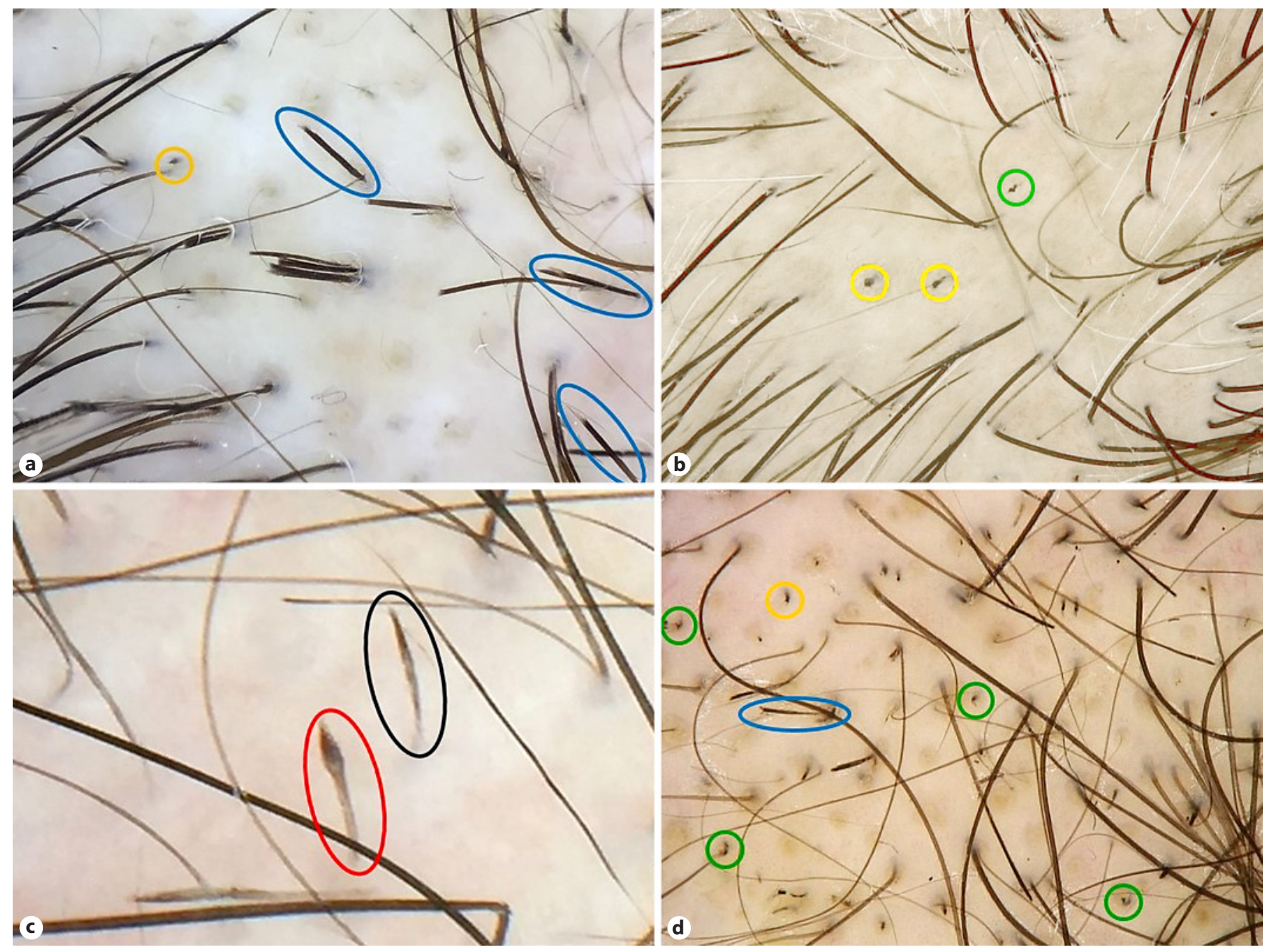

Fig. 4. a-d Trichoscopic features of trichotillomania, showing trichoptilosis (blue circles), black dots (yellow circles), flame hairs (green circles), tulip hair (red circle), and micro-exclamation mark hair (black circle) $(\times 20)$.

on its own, and parental education and support is usually enough to control the symptoms. However, in older children and adolescents, behavioral approaches combined with pharmacotherapy offer more clinical benefits due to the high incidence of associated psychiatric disorders [10].

\section{Nonpharmacological Treatments}

Significant benefits are usually obtained with cognitive-behavioral therapy (CBT), currently the most empirically validated treatment option [9]. Habit reversal training (HRT), a type of CBT, involves helping patients acquire awareness of their hair-pulling behavior and then replacing that behavior with other activities that patients can perform with their hands whenever they feel the urge to pull out their hair [4, 9-11].
Recently, the invention of electronic devices that monitor habits may improve the effectiveness of HRT. One example device unit consists of an electronic necklace that casts inaudible sound waves around the head and communicates to a bracelet that vibrates when crossing into the head region for longer than $3 \mathrm{~s}$. This type of technology may present an opportunity to target therapy at the unconscious pulling predominantly seen in the pediatric population [4]. Stimulus control training is a technique that modifies the person's environment to make it less favorable to hair-pulling behavior [10].

Other psychotherapy methods include dialectical behavioral therapy, exposure and ritual prevention therapy, metacognitive therapy, acceptance and commitment therapy, and support group therapy [10]. Support groups 
Table 1. Diagnostic criteria for trichotillomania according to the Diagnostic and Statistical Manual of Mental Disorders

DSM-V diagnostic criteria for trichotillomania

Recurrent pulling out of one's hair, resulting in hair loss

Repeated attempts to decrease or stop hair pulling

The hair pulling causes significant distress or impairment in social or occupational functioning

The hair pulling or hair loss is not attributable to another medical condition

The hair pulling is not better explained by the symptoms of another mental disorder

are common in many medical conditions to bring together individuals to sharing challenges and successes of their illness management. A small study comparing group behavioral therapy to supportive therapy in adults demonstrated short-term improvement in those in group behavioral therapy, suggesting that this could play an adjunctive role in trichotillomania [4].

Alternative behavioral intervention approach, such as hypnotherapy, has been utilized to sensitize and alert patients to impending pulling behaviors. The lack of controlled trials of hypnotherapy makes it difficult to ascertain the true efficacy of this therapy [4].

\section{Pharmacological Treatment}

No medications are specifically approved for the treatment of trichotillomania. However, some drugs, like selective serotonin reuptake inhibitors (SSRIs) and the tricyclic antidepressant clomipramine, may help control the symptoms. It has likely more to do with psychiatric comorbidity than the efficacy for trichotillomania itself $[5,10]$.

\section{Tricyclic Antidepressant}

Clomipramine blocks the reuptake of norepinephrine and serotonin and also blocks muscarinic cholinergic, adrenergic, $\mathrm{H} 1$, and 5HT2 receptors [10]. Studies showed successful responses in clomipramine monotherapy at $125 \mathrm{mg} /$ day and dual therapy at $50 \mathrm{mg} /$ day combined with behavioral therapy. When compared with CBT and placebo, clomipramine was significantly less effective than CBT but showed more benefits than placebo [10]. Adverse effects are relatively frequent and include dry mouth, constipation, drowsiness, and sedation $[10,13]$.

\section{Selective Serotonin Reuptake Inhibitors}

Although SSRIs are the most often chosen treatment option for trichotillomania, evidence of benefit is weak. Studies comparing fluoxetine and placebo in patients with trichotillomania showed no significant differences in reducing the urge to tear hairs, time spent on pulling hairs, and number of torn hairs [9].

A small study showed benefit with doses of up to 80 mg daily. However, since other psychiatric conditions such as depression and anxiety are common in patients with trichotillomania, and SSRIs have shown efficacy in these conditions, it is reasonable to include this medication in the patient's regimen [10].

\section{Antipsychotics}

Olanzapine is the most studied antipsychotic for the treatment of trichotillomania. Although dosages of 2.5$10 \mathrm{mg}$ daily revealed significant improvement, olanzapine has many side effects, including metabolic dysfunction and extrapyramidal symptoms, which should be weighed against potential benefit [9].

\section{N-Acetylcysteine}

NAC is a modulator of glutamic acid action, which has been recently used in psychodermato logical conditions such as onychotillomania, skin-picking disorders, prurigo, trichoteiromania, and trichotillomania $[9,10,12]$. The mechanism of action of NAC for neurologic and psychiatric disorders is not fully understood, but it is possibly related to its action on the glutamate system. Glutamatergic hyperactivity leading to excitotoxicity and oxidative stress has been implicated in the pathogenesis of impulse control disorders, such as trichotillomania. The decrease in glutamate levels, being the main excitatory neurotransmitter in the nucleus accumbens, would explain NAC efficacy in controlling compulsive behavior [10, 12].

While NAC demonstrated significant effect in adults with trichotillomania using $1,200 \mathrm{mg}$ twice daily, data from the pediatric trial did not show any benefit $[4,10]$. Possibly, children do not respond to NAC as adults react because children are more likely to engage predominantly in an automatic pattern of pulling, and NAC may be better for focused pulling by reducing urges to pull hair [13]. Due to its relative safety and tolerability compared 
to other pharmacological treatments for trichotillomania, NAC has the potential to be an important option, especially for refractory cases $[4,10]$.

\section{Opioid Antagonists}

Naltrexone is the opioid antagonist most studied for the treatment of trichotillomania. It reduces the dopamine levels in the nucleus accumbens, which seems to be involved in the brain's reward pathway [10]. It showed effectiveness in trichotillomania in some case reports by reducing the hair-pulling urge. However, in a doubleblind randomized controlled trial using naltrexone 150 $\mathrm{mg}$ daily, there was no significant difference in reducing hair pulling between drug and placebo $[9,10]$.

\section{Dronabinol}

Dronabinol is a cannabinoid agonist that seems to have potential benefits in the treatment of trichotillomania by diminishing glutamate cytotoxicity in the striatum. In a small study, using dronabinol at the dosage of $2.2-15 \mathrm{mg} /$ day, the drug showed significant benefits in reducing hairpulling behavior in $75 \%$ of the subjects. Furthermore, at this dosage, the medication was generally well tolerated, with no significant deleterious effects on cognition $[9,10]$.

\section{Conclusion}

Trichotillomania is a poorly understood disorder that can be extremely disabling with evident impact on quality of life and social and psychological functioning of affected patients. Factors that suggest progression include an increase in the reported number of pulling sites, frequency of urges, and amount of focused pulling.
Trichotillomania requires a comprehensive treatment plan and interdisciplinary approach. During the treatment process, the physician should always have a nonjudgmental, empathic, and inviting attitude toward the patient. The role of psychiatry-dermatology liaison is essential in addition to patient and parent support services [2]. The provider should educate the patient and their family, highlighting psychosocial effects and triggers, discussing available treatment options, among which CBT is currently the firstline treatment, especially in children [4].

\section{Statement of Ethics}

Written informed consent was obtained from the patients for publication of any accompanying images.

\section{Conflict of Interest Statement}

Dr. Tosti reports being a consultant - DS Laboratories, Monat Global, Almirall, Tirthy Madison, Eli Lilly, Bristol Myers Squibb, and $\mathrm{P} \& \mathrm{G}$.

\section{Funding Sources}

This research did not receive any specific grant from funding agencies in the public, commercial, or not-for-profit sectors.

\section{Author Contributions}

A.T., D.F.M., and C.D.L. conceived the study. D.F.M. and C.S.L. wrote the manuscript. A.T. and B.M.P. reviewed the final manuscript.

\section{References}

1 Fernandes MRN, Melo DF, Vincenzi C, Lima CDS, Tosti A. Trichotillomania incognito: two case reports and literature review. Skin Appendage Disord. 2021;7(2):131-4.

2 França K, Kumar A, Castillo D, Jafferany M, Hyczy da Costa Neto M, Damevska K, et al. Trichotillomania (hair-pulling disorder): clinical characteristics, psychosocial aspects, treatment approaches, and ethical considerations. Dermatol Ther. 2019; Jul;32(4):e12622.

3 Grant JE, Chamberlain SR. Trichotillomania. Am J Psychiatry. 2016 Sep1;173(9):868-74.

4 Henkel ED, Jaquez SD, Diaz LZ. Pediatric trichotillomania: review of management. Pediatr Dermatol. 2019 Nov;36(6):803-7.

5 Martín JM, Montesinos E, Cordero P, Gonzalez V, Ramon D. Trichoscopy features of trichotillomania. Pediatr Dermatol, 2019 Mar;36(2):265-7.

6 Woods DW, Houghton DC. Diagnosis, evaluation, and management of trichotillomania. Psychiatr Clin North Am. 2014 Sep;37(3):301-17.

7 Stefanato CM. Histopathology of alopecia: a clinicopathological approach to diagnosis. Histopathology. 2010 Jan;56(1):24-38.

8 Lacarrubba F, Micali G, Tosti A. Scalp dermoscopy or trichoscopy. Curr Probl Dermatol. 2015;47:21-32.

9 Cison H, Kus A, Popowicz E, Szyca M. Trichotillomania and trichophagia: modern diagnostic and therapeutic methods. Dermatol Ther. 2018 Sep;8(3):389-98.

10 Everett GJ, Jafferany M, Skurya J. Recent advances in the treatment of trichotillomania (hair-pulling disorder). Dermatol Ther. 2020 Nov;33(6):e13818.

11 Trüeb R, Gieler U. Psychocutaneous disorders of hair and scalp. In: Blume-Peytavi $U$, Tosti A, Whiting DA, Trüeb R, editors. Hair growth and disorders. 1st ed Berlin: Springer; 2008. p. 414-7.

12 Salas-Callo CI, Pirmez R. Trichoteiromania: good response to treatment with n-acetylcysteine. Skin Appendage Disord. 2019;5(4): 242-5.

13 Farhat LC, Olfson E, Nasir M, Levine JLS, Li F, Miguel EC, et al. Pharmacological and behavioral treatment for trichotillomania: an updated systematic review with meta-analysis. Depress Anxiety. 2020 Aug;37(8):71527. 